\title{
Dynamic Contracts and Learning by Doing*
}

\author{
Julien Prat ${ }^{\dagger}$ \\ This Version: August 2014 (First Version: January 2014)
}

\begin{abstract}
This paper studies the design of optimal contracts in dynamic environments where agents learn by doing. We derive a condition under which contracts are fully incentive compatible. A closed-form solution is obtained when agents have CARA utility. It shows that human capital accumulation strengthens the power of incentives and allows the principal to provide the agent with better insurance against transitory risks.
\end{abstract}

*I thank Boyan Jovanovic for his invaluable support and advice. I am grateful to an anonymous referee and to seminar participants at the University of Zurich, CERGE-EI and Federal Reserve of Minneapolis for comments and suggestions. I acknowledge the support of the Labex Ecodec (ANR-11-LABX-0047) and of the Barcelona GSE.

${ }^{\dagger}$ CNRS, CREST, UMR2773, Paris; Institute for Economic Analysis (CSIC), BGSE, Barcelona. Email: julien.prat@ensae.fr. 


\section{Introduction}

In most jobs, productivity depends as much on training as on effort. Young workers may be highly motivated, they will nonetheless find it difficult to match the efficiency of their experienced colleagues. As the saying goes, practice makes perfect. The importance of learning-by-doing has long been recognized in the literature on human capital. ${ }^{1}$ Yet, its implications for agency problems remain largely unexplored. Is it necessary to link wages more tightly to performances when the agent's action determines her future productivity? More generally, is human capital accumulation reinforcing or weakening the power of incentives?

A formal treatment of these questions has been hindered by the fact that effort has an impact on the stock of human capital, and so matters for the distribution of output in future periods. When actions are hidden, a deviation generates persistent private information. Consider, for example, an agent that provides less effort than expected. Not only will her productivity today be lower than anticipated by the principal, but she will also carry less skills into next period. In other words, the agent will be less optimistic than the principal about her future prospects.

This is why excluding one shot deviations is not enough to establish whether or not a contract is incentive compatible. Due to the persistent effect of past actions, one also has to exclude multiple deviations. A general treatment of the problem would therefore involve keeping track of all the possible strategies. But more often than not, such a direct approach is impractical as the state space quickly becomes unbounded. ${ }^{2}$

We circumvent these difficulties by using a continuous time model and by establishing full incentive compatibility. We focus on the conjectured equilibrium and characterize the agent's first-order condition. Then we derive a sufficient condition under which a contract satisfying the first-order condition is also fully incentive compatible. In other words, we identify a requirement such that multiple deviations are never profitable when local deviations are suboptimal. The sufficiency condition ensures that the solution of the relaxed problem subject to the first-order constraint is also a solution of the original incentive problem.

\footnotetext{
${ }^{1}$ See the seminal work of Becker (1964).

${ }^{2}$ Fernandes and Phelan (2000) propose a framework where the state space remains manageable. They assume that output is only correlated from one period to the next, and that agents can take one of only two actions. Then the relevant deviation is unique, and it is sufficient to keep track of a single "threat keeping constraint".
} 
We characterize the necessary and sufficient conditions for general time-separable preferences. An action satisfies the first-order condition when its marginal cost is equal to the current pay-performance sensitivity plus the appropriately discounted sum of future sensitivities. The first component is standard and corresponds to the solution in Sannikov (2008) where the agent cannot accumulate human capital. Specific to our problem is the addition of future sensitivities: Higher effort increases the stock of human capital which raises expected output in future periods. From the principal's standpoint, this creates a series of output surprises whose returns in utils are by definition proportional to the sensitivity coefficients. Since performance and pay are positively correlated, it follows that the value of private information is also positive. This is why learning-by-doing strengthens the power of incentives by relaxing the incentive constraint. The agent adds the benefits of higher skills in the future to the current returns, which makes her more eager to provide effort.

In order to go further than the description of the incentive constraints, we analyze the optimal contract when agents have CARA utility. This specification allows us to solve for the optimal contract in closed form because it neutralizes the wealth effect. The solution confirms the insights gathered from the analysis of the first-order condition: Human capital accumulation lowers the cost of incentives provision, thereby reducing the distance between the Pareto frontier and the first-best allocation. The principal is able to offer better insurance so that wages becomes less tightly linked to performances when the agent learns by doing.

Related literature. This paper is closely related to the companion article by Jovanovic and Prat (2013) which studies incentive contracts when the agent's type is gradually revealed over time. ${ }^{3}$ The learning process also generates persistent private information because the agent can manipulate the principal's beliefs by providing a different level of effort than expected. In contrast with learning-by-doing, the value of private information is negative: Following a positive deviation from the recommended action, the agent becomes less optimistic about her type than the principal. This is why learning weakens the power of incentives whereas human capital strengthens it. DeMarzo and Sannikov (2011) also introduces learning into a dynamic moral hazard problem but they assume that both parties are risk neutral, whereas agents are risk averse in our model. Hence their paper

\footnotetext{
${ }^{3} \mathrm{He}$ et al. (2012) extends Jovanovic and Prat (2013) by introducing hidden savings and effort costs which are convex instead of linear.
} 
bypasses the insurance-incentive tradeoff at the heart of our analysis, and focuses instead on the risk of inefficient termination of the relationship.

More broadly, this paper belongs to a burgeoning strand of research which uses continuous time techniques to study the design of dynamic incentives in environments featuring persistent information. The seminal work on the issue is Williams (2011). It studies a reporting problem with a persistent hidden state. Williams also uses a first-order approach but our proof differs: Whereas Williams' approach relies on the stochastic maximum principle, we use instead a variational argument originally proposed by Cvitanić et al. (2009). Aside from this methodological contribution, the mechanisms at work in our model is fundamentally different. In Williams (2011), the agent can manipulate the principal's belief about her type by misreporting it, but the agent's action does not affect her future productivity. $^{4}$

We are attributing persistency to human capital accumulation, but there are other valid interpretations. Given the partial equilibrium nature of our analysis, nothing prevents reader from favoring alternative mechanisms. For example, one may think of the long-run impact that CEO's actions have on firm performance. This is precisely the issue addressed by Sannikov (2012) in his concurrent research about long-run incentives. Aside from deriving similar constraints, Sannikov proposes to replace the original state variables by their Lagrange multipliers. He uses this approach to characterize contracts when the agent's utility is separable in consumption and leisure. ${ }^{5}$ The model shows that optimal payments can be postponed beyond the termination of the relationship.

Outline of the paper. The structure of the problem is described in Section 2. We present the first-order approach in Section 3 where we derive both necessary and sufficient conditions. In Section 4, we restrict our attention to agents with CARA utility. We derive the principal's value function in closed-form and characterize the dynamics of wages. Section 5 concludes while the proofs of the Propositions and Corollaries are relegated to the Appendix.

\footnotetext{
${ }^{4}$ The companion paper Williams (2013) studies a dynamic moral hazard problem where the agent has access to states which the principal cannot observe. As an illustration of his general results, Williams (2013) solves a model where the hidden states corresponds to the amount of savings accumulated by the agent.

${ }^{5}$ By contrast, our closed form example uses a utility function that is not separable in consumption and leisure.
} 


\section{The Contracting Problem}

Technology. - The agent's effort $a_{t} \in \mathcal{A} \subset \mathbb{R}^{+}$has two effects. First, it raises output in the current period. This immediate benefit is followed by a permanent one as agents learn by doing. Effort adds to the stock of human capital $h$ which evolves as follows

$$
d h_{t}=\left(a_{t}-\delta h_{t}\right) d t, \text { with } \delta \geq 0 .
$$

This specification accounts for the natural obsolescence of skills since $h$ decreases at the rate $\delta$. In order to offset the depreciation of her human capital, the agent has to provide some effort whose return in units of human capital is assumed to be linear. Thus the cumulative output $Y_{t}$ produced by the agent up to time $t$ obeys the following dynamic

$$
Y_{t}=\int_{0}^{t}\left(a_{s}+h_{s}\right) d s+\int_{0}^{t} \sigma d Z_{s}
$$

where $Z_{t}$ is a standard Brownian Motion. Productivity is given by the sum of effort plus the stock of human capital. ${ }^{6}$ The agent knows both her action and skill level. By contrast, the principal only observes output and the noisiness of the technology prevents him from observing the action taken by the agent as well as her skill level.

Contract.-We analyze long-term contracts that last until date $T$ and whose payments $w_{t}$ can depend on the output history $\left\{Y_{s} ; s \in[0, T]\right\}$ in an arbitrary way. More formally, let $(\Omega, \mathcal{F}, P)$ denote the probability space on which the Brownian motion $Z$ is defined. Since output paths are random elements of the space $\Omega, w:[0, T] \times \Omega \rightarrow \mathbb{R}$ is a mapping that associates a wage to any event $\omega \in \Omega$. The information available to the principal does not include effort. It is therefore restricted to the filtration $\mathcal{F}_{t}^{Y} \triangleq \sigma\left(Y_{s} ; 0 \leq s \leq t\right)$ generated by $Y$, whose augmentation we denote $\mathbb{F}^{Y} \triangleq\left\{\mathcal{F}_{t}^{Y}\right\}_{t \geq 0}$, and so the mapping $w$ must be $\mathbb{F}^{Y}$-measurable.

Preferences. - The agent chooses her effort and receives the flow utility $u\left(w_{t}, a_{t}\right)$ in return. We assume that the instantaneous utility function $u(w, a) \in \mathcal{C}^{2,2}(\mathbb{R} \times \mathcal{A})$. Preferences are time additive with discount rate $\rho>0$ so that the agent's preferences as of time

\footnotetext{
${ }^{6}$ It is straightforward to generalize the production function by adding a multiplicative term $\alpha \in[0,1]$ in front of effort, i.e., $Y_{t}=\int_{0}^{t}\left(\alpha a_{s}+h_{s}\right) d s+\int_{0}^{t} \sigma d Z_{s}$. All the derivations presented in this paper go through under this more general specification. Notice that Sannikov (2012) focuses on cases where $\alpha=0$ so that effort affects output solely through its impact on the stock variable $h$.
} 
0 read

$$
\mathcal{U}_{0} \triangleq \int_{0}^{T} e^{-\rho t} u\left(w_{t}(\omega), a_{t}\right) d t+e^{-\rho T} U\left(W_{T}(\omega)\right),
$$

with terminal utility $U(W) \in \mathcal{C}^{1}(\mathbb{R})$. The principal is risk neutral and seeks to maximize the discounted flow of output net of wages and terminal payment. The optimal contract $w$ and agent's strategy $a$ are therefore chosen so as to optimize the principal's expected profit

$$
\pi_{0} \triangleq E^{a}\left[\int_{0}^{T} e^{-\rho t}\left(d Y_{t}-w_{t}\right) d t-e^{-\rho T} W_{T}(\omega)\right],
$$

under the expectation $E^{a}$ associated to the agent's strategy. Maximization is performed subject to the participation constraint at time 0 , i.e., $E^{a}\left[\mathcal{U}_{0}\right] \geq \underline{\mathcal{U}}$ for an exogenously given $\underline{\mathcal{U}}$; and to the incentive constraint $\underline{\mathcal{U}} \geq E^{\hat{a}}\left[\mathcal{U}_{0}\right]$ for all feasible strategies $\hat{a}$. The incentive constraint is too general to be handled analytically. We explain in the following section how it implies a necessary condition that can be used to characterize incentive compatible strategies.

\section{Incentive Constraints}

We use a first-order approach to determine whether strategies are incentive compatible. We assume that the agent has not deviated in the past and derive a necessary condition under which local deviations cannot be optimal. Then we generalize the analysis to arbitrary strategies and obtain a sufficient condition which can be combined with the necessary condition to establish optimality, under both local and global deviations. This second step is crucial because the stock of human capital depends on past actions. Because of this persistence, excluding local deviation does not ensure that agents will not use multiple deviations.

\section{$3.1 \quad$ Necessary condition}

The agent chooses her effort so as to maximize her continuation value

$$
v_{t} \triangleq \sup _{a_{t} \in \mathcal{A}} E_{t}^{a}\left[\int_{t}^{T} e^{-\rho(s-t)} u\left(w_{s}, a_{s}\right) d s+e^{-\rho(T-t)} U\left(W_{T}\right)\right],
$$


As explained before, the earning process $w_{t}$ depends on the whole output path and is therefore non-Markovian. The optimal control of non Markovian processes can be analyzed using a martingale approach. Intuitively, one can think of the agent as controlling the distribution of outputs, and consequently wages, through his choice of effort. This is formally equivalent to choosing the probability measure over realizations of $w_{t}$ and has the advantage that the Radon-Nikodym derivative associated to a given effort path is Markovian, which renders the problem amenable to standard optimization techniques.

Proposition 1 There exists a unique decomposition of the agent's continuation value

$$
\begin{aligned}
d v_{t} & =\left[\rho v_{t}-u\left(w_{t}, a_{t}\right)\right] d t+\gamma_{t} \sigma d Z_{t} \\
v_{T} & =U\left(W_{T}\right)
\end{aligned}
$$

where $\gamma$ is a square integrable process. The necessary condition for $a_{t}$ to be an optimal control reads

$$
\left[\gamma_{t}+E_{t}^{a}\left[\int_{t}^{T} e^{-(\rho+\delta)(s-t)} \gamma_{s} d s\right]+u_{a}\left(w_{t}, a_{t}\right)\right]\left(\hat{a}-a_{t}\right) \leq 0
$$

for all $\hat{a} \in \mathcal{A}$.

When effort is interior and the necessary condition binds, (6) is equivalent to

$$
-u_{a}\left(w_{t}, a_{t}\right)=\gamma_{t}+E_{t}^{a}\left[\int_{t}^{T} e^{-(\rho+\delta)(s-t)} \gamma_{s} d s\right]
$$

which basically ensures that marginal costs and marginal returns are identical. The marginal costs of an additional unit of effort is equal to $-u_{a}\left(w_{t}, a_{t}\right)$. Its benefits are twofold. First, the technology of production is such that effort raises the output flow one for one. The benefit in utils of such an increase is by definition equal to $\gamma_{t}$, since it measures the sensitivity of the promised value with respect to output. There is also a second effect due to the persistent impact that effort has on the stock of human capital. One more unit of effort today raises the $\tau$-periods ahead value of $h$, and thus output, by $\exp (-\delta \tau)$. As for the future benefits, their values in utils are obtained multiplying the output surprise by the expected value of $\gamma$. Discounting all these future gains at rate $\rho$ and summing them, one obtains the integral on the right hand side of (7).

To see that human capital strengthens the power of incentives, it is instructive to let $\delta$ diverges to infinity. Then agents cannot accumulate any human capital because their 
stock of knowledge immediately depreciates. The integral in (7) converges to zero and we recover the standard incentive constraint in Sannikov (2008). ${ }^{7}$ The integral converges to zero from above because incentives can be positive solely if $\gamma>0$. It follows that in order to implement a given effort $a_{t}$ at a given wage $w_{t}$, the sensitivity coefficient $\gamma_{t}$ with full depreciation must be higher. In other words, contracts without human capital accumulation link pay more tightly to performance, which lowers the welfare of the riskaverse agent. $^{8}$

\subsection{Sufficient conditions}

We focus on contracts that fulfill the necessary condition (6). The first-order approach is justified solely if the resulting solution satisfies the general incentive constraints, that is if it rules out both local and global deviations. This is not guaranteed in our setup because any departure from expected effort drives a permanent wedge between the expectations of the agent and that of the principal. The following proposition provides a sufficient condition under which a contract satisfying the relaxed constraint (6) is indeed fully incentive compatible.

Proposition 2 Let $p_{t} \triangleq E_{t}^{a}\left[\int_{t}^{T} e^{-(\rho+\delta)(s-t)} \gamma_{s} d s\right]$ denote the value of private information. It admits a unique decomposition

$$
\begin{aligned}
d p_{t} & =\left[(\rho+\delta) p_{t}-\gamma_{t}\right] d t+\vartheta_{t} \sigma d Z_{t} \\
p_{T} & =0
\end{aligned}
$$

where $\vartheta$ is a square integrable process. A control $a_{t}$ is incentive compatible if (6) and

$$
2 u_{a a}\left(w_{t}, a_{t}\right) \leq \vartheta_{t}
$$

are true for almost all $t \in[0, T]$.

\footnotetext{
${ }^{7}$ Our incentive constraint is also closely related to the one in Sannikov (2012) which reads $-u_{a}(\cdot)=$ $E_{t}^{a}\left[\int_{t}^{T} e^{-\rho(s-t)} f(s-t) \gamma_{s} d s\right]$. The function $f(s-t)$ captures the effect of past action $a_{s}$ on the value at time $t$ of the stock variable. It is therefore equal to $\exp (-\delta(s-t))$ in our model. More importantly, the volatility coefficient $\gamma_{t}$ does not anymore appear on the right hand side of the incentive constraint. This is because, as discussed in footnote 6, Sannikov (2012) assumes that effort has no direct effect on output, i.e., $Y_{t}=\int_{0}^{t} h_{s} d s+\int_{0}^{t} \sigma d Z_{s}$.

${ }^{8}$ The argument is mostly heuristic because both wages and effort are endogenous. Yet, we will see that its intuition is valid when we solve for the optimal contract under CARA utility.
} 
Condition (10) is sufficient but not necessary: A contract might violate it and be nonetheless fully incentive compatible. It cannot be checked before solving for the contract since $w_{t}, a_{t}$ and $\vartheta_{t}$ are all endogenous. But one can use (10) ex-post, that is once a contract satisfying the relaxed condition (6) has been identified. We show in the next section how the whole procedure works when the agent's utility function is CARA.

\section{Incentive Contracts under CARA Utility}

We assume that the agent's per-period utility is CARA

$$
u(w, a)=-\exp (-\theta(w-\lambda a)), \text { with } \lambda \in(0,1), \theta>0,
$$

and $a \in \mathcal{A}=(0,1]$. CARA utility functions have the advantage of ruling out wealth effects. The parameter $\theta$ measures the degree of risk aversion exhibited by the agent, while the requirement that $\lambda<1$ ensures that maximal effort $a=1$ is also the first best action. In order to focus on incentive providing contracts, we exclude 0 from the set $\mathcal{A}$ of implementable actions. We introduce later the possibility to retire the agent and derive the condition under which it is optimal to terminate incentives provision.

For tractability, we let the contracting horizon $T$ diverge to infinity. This allows us to derive a stationary value function which does not directly depend on time. The solution holds under the premise that the two state variables satisfy standard transversality conditions. We also propose in Appendix B a constructive approach: We solve the problem for finite $T$, and prove that the infinite horizon solution is the limit of a sequence of relaxed problems in finite horizon that satisfy the sufficiency condition (10). ${ }^{9}$

The principal's problem reads

$$
\pi_{t}=\max _{\{a, w, \gamma, \vartheta\}} E_{t}\left[\int_{t}^{\infty} e^{-\rho(s-t)}\left(a_{s}+h_{s}-w_{s}\right) d s\right]
$$

subject to the incentive constraint

$$
-u_{a}\left(w_{t}, a_{t}\right)=\gamma_{t}+p_{t}
$$

\footnotetext{
${ }^{9}$ This constructive approach shows that the solution in Proposition 3 is not invalidated by the lack of mutually absolutely continuous measure in the infinite horizon limit.
} 
and the laws of motion of the state variable ${ }^{10}$

$$
\begin{aligned}
d v_{t} & =\left[\rho v_{t}-u\left(w_{t}, a_{t}\right)\right] d t+\gamma_{t} \sigma d Z_{t} \\
d p_{t} & =\left[(\rho+\delta) p_{t}-\gamma_{t}\right] d t+\vartheta_{t} \sigma d Z_{t}
\end{aligned}
$$

along with their transversality conditions, $\lim _{s \rightarrow \infty} E_{t}^{a}\left[e^{-\rho s} v_{s}\right]=\lim _{s \rightarrow \infty} E_{t}^{a}\left[e^{-(\rho+\delta) s} p_{s}\right]=$ 0 . We relegate the derivations to the Appendix and report the expression of the expected wage bill in the following Proposition.

Proposition 3 It is optimal to set $a_{t}=1$ for all $t$. The expected wage bill of the optimal contract can be expressed as a function of the promised value $v$ only. It reads

$$
\rho B(v) \triangleq \rho E_{t}^{a}\left[\int_{t}^{\infty} e^{-\rho(s-t)} w_{s} d s\right]=\lambda-\frac{\ln (-\rho v)}{\theta}-C .
$$

The constant $C$ is equal to

$$
C=\frac{\ln (k / \rho)}{\theta}+\frac{\rho-k}{\rho \theta}-\frac{(\sigma \lambda)^{2} \theta}{2 \rho}\left[k\left(\frac{k+\delta}{k+\delta+1}\right)\right]^{2},
$$

where $k$ is the unique positive root of the cubic equation

$$
k^{3}(\theta \lambda \sigma)^{2}+k^{2}\left[(\theta \lambda \sigma)^{2} \delta+1\right]+k(\delta+1-\rho)-\rho(\delta+1)=0
$$

To see why the expected wage bill does not depend on $p$, replace the incentive constraint into the law of motion (12) to obtain

$$
d p_{t}=\left[(\rho+\delta+1) p_{t}+u_{a}\left(w_{t}, a_{t}\right)\right] d t+\vartheta_{t} \sigma d Z_{t}
$$

The state variable $p$ is therefore equivalent to

$$
p_{t}=-E_{t}^{a}\left[\int_{t}^{\infty} e^{-(\rho+\delta+1)(s-t)} u_{a}\left(w_{s}, a_{s}\right) d s\right] \text {. }
$$

Under our CARA specification, marginal and total utilities are proportional as $u_{a}(\cdot)=$ $\theta \lambda u(\cdot)$. We show in the proof of Proposition 3 that this property ensures that the ratio $p_{t} / v_{t}$ remains constant over time. This is why the promised value $v$ encapsulates all the

\footnotetext{
${ }^{10}$ In Williams' (2011) model, the variable $p$ corresponds to the co-state associated to the variable $z$ capturing the covariation between reports and the stock of lies.
} 
relevant information.

Expected profits $\pi$ directly follow from the wage bill as

$$
\rho \pi\left(h_{t}, v_{t}\right)=\rho E_{t}^{a}\left[\int_{t}^{\infty} e^{-\rho(s-t)}\left(h_{s}+a_{s}-w_{s}\right) d s\right]=\frac{\rho h_{t}+1}{\rho+\delta}+1-\rho B\left(v_{t}\right) .
$$

Given that effort remains equal to 1 , the stock of human capital $h_{t}=\left(1-e^{-\delta t}\right) / \delta$ is deterministic and gradually converges to its steady-state level $\bar{h} \triangleq \lim _{t \rightarrow \infty} h_{t}=1 / \delta$. By contrast, the expected wage bill evolves stochastically so as to satisfy the promise keeping constraint. To understand the intuition behind (13), consider first the term involving the promised value: $\lambda-\ln (-\rho v) / \theta$. Given the specification (11) of the utility function, the cost of delivering value $v$ through a constant income stream is equal to $-\ln (-\rho v) / \theta$, whereas $\lambda$ compensates the disutility incurred by the agent for providing $a=1$. Hence, $\lambda-\ln (-\rho v) / \theta$ is the prevailing wage in first best environments where the principal can perfectly insure the agent. Thus $C$ measures the flow costs of incentive provision or, in other words, the loss induced by the information problem. Perhaps surprisingly, the loss does not depend on the level of the promised value. This feature is due to the CARA specification and its lack of wealth effect, so that incentives provision does not become costlier as the agent gets richer.

The constant $C$ depends on the root of a cubic equation. Thus it is not immediately clear how it varies with the model's parameters. We show in the Appendix that the root $k$ always exists, is unique and decreasing in the rate of decay of human capital $\delta$. As $C$ is increasing in $k$, Corollary 1 immediately follows.

Corollary 1 The expected wage bill $B(v)$ is increasing in $\delta$.

The less persistent human capital is, the higher the costs of motivating the agent. In particular, it is always more expensive to induce full effort when there is no human capital accumulation, i.e., when $\delta$ diverges to infinity. This is in line with our discussion of the first order condition (7) at the end of Section 3.2: Learning-by-doing relaxes the incentive constraint because agents add future output gains to the immediate benefit of effort provision.

Figure 1 illustrates our finding. It depicts the per-period costs $C$ of incentive provision as a function of $\delta$ and $\sigma$. The costs are increasing in output volatility $\sigma$ because it lowers the signal/noise ratio. This makes it more difficult to identify shirkers which raises the costs of eliciting effort. The information problem vanishes as $\sigma$ goes to zero because the principal can perfectly infer the actual level of effort. Since $C$ measures the distance from 


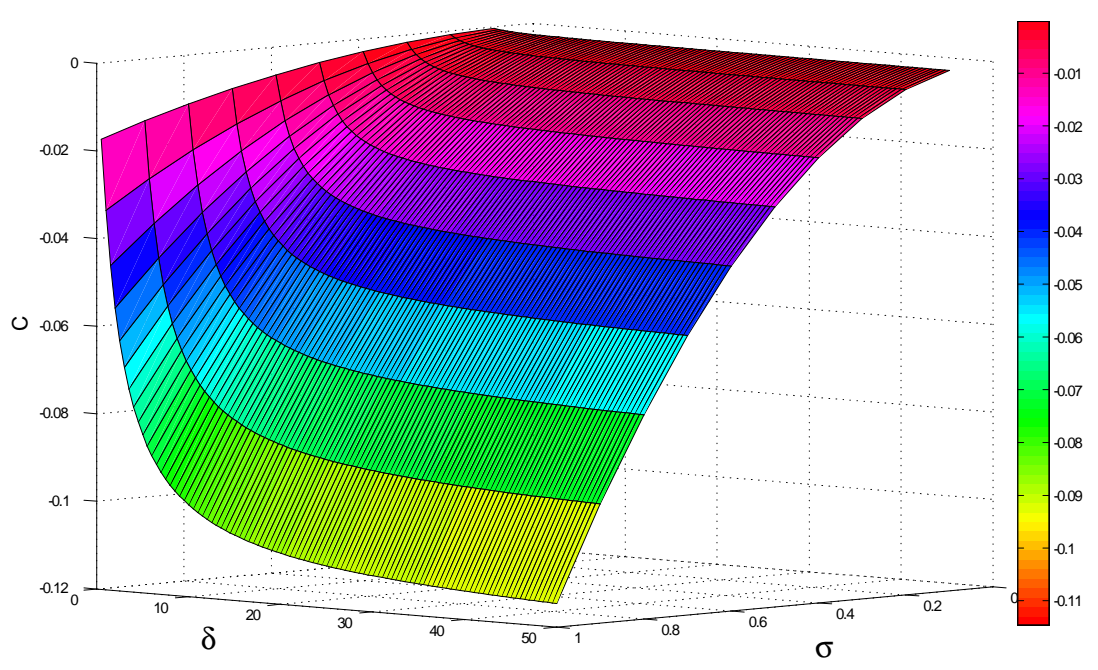

Figure 1: Costs of Incentive Provision. Parameters: $\rho=0.1, \lambda=0.5, \sigma=1, \theta=10$.

the first best contract, its value converges to zero with $\sigma$. Turning our attention to $\delta$, we see that Corollary 1 is indeed confirmed by the simulation: Losses are unambiguously increasing in $\delta$.

Wage dynamics. - It is instructive to analyze how wages evolve over time in order to understand why the cost of delivering a given promised value is decreasing in the persistence of human capital. It is shown in the proof of Proposition 3 that the sensitivity of the promised value reads ${ }^{11}$

$$
\gamma_{t}=-\frac{1}{\theta \lambda \sigma^{2}}\left(\frac{\rho}{k}-1\right) v_{t}
$$

Since $\partial k / \partial \delta<0$, it is clear that $\partial \gamma / \partial \delta>0$. The less persistent human capital is, the more volatile the continuation value. This is why the expected wage bill has to be higher in order to compensate the agent for her greater exposure to risk.

We can also characterize the effect that $\delta$ has on the drift of $v$. Reinserting the wage $w(v)=-\ln (-k v) / \theta+\lambda$ into (4), we find that

$$
d v_{t}=v_{t}(\rho-k) d t+\gamma_{t} \sigma d Z_{t}
$$

\footnotetext{
${ }^{11}$ The sensitivity coefficient $\gamma$ is unambiguously positive because $v$ is negative and, as shown in the proof of Corollary $1, \rho>k$.
} 
The trend is negative ${ }^{12}$ which shows that the principal finds it optimal to frontload transfers. This process of immiserization is common in models of full commitment with CARA utility. More interestingly, the drift coefficient is decreasing in $\delta$ as $\partial k / \partial \delta<0$. Learningby-doing counteracts the agent's immiserization. It become more profitable to backload payments when actions have a persistent effect because agents take these future benefits into account, which relax the incentive constraint. However, since $\rho$ is superior to $k$ even when $\delta=0$, the immiserization channel always dominates. ${ }^{13}$

In order to derive similar insights for wages, we apply Ito's lemma to $w(v)=-\ln (-k v) / \theta+$ $\lambda$ and find that $w_{t}$ obeys

$$
d w_{t}=\frac{1}{\theta}\left[\left(k-\rho+\frac{1}{2(\theta \lambda \sigma)^{2}}\left(\frac{\rho}{k}-1\right)^{2}\right) d t+\frac{1}{\theta \lambda \sigma}\left(\frac{\rho}{k}-1\right) d Z_{t}\right] .
$$

As expected, wages fluctuate more when $v$ is volatile, that is when $\delta$ is higher. By contrast, the impact of $\delta$ on the deterministic component of the wage schedule turns out to be ambiguous. As discussed above, human capital persistence creates some backloading which directly raises the coefficient of the drift. But it also lowers the volatility of $v$ and, since wages are convex in $v$, this puts some downward pressure on wages. The simulation reported in Figure 2 illustrates that, for some parameter values, the drift of $w$ can be increasing in $\delta$ because of the volatility channel.

Full Incentive Compatibility. - We have used the relaxed incentive problem to derive optimal contracts. We still have to establish that they satisfy, not only the first order condition, but also the global incentive constraint.

Corollary 2 The contract described in Proposition 3 is fully incentive compatible because it satisfies both the necessary condition (7) and sufficient condition (10).

One should expect that full incentive compatibility is ensured for high enough $\delta$ because the model converges to a standard contracting problem without persistence. Then we know from the work of Schättler and Sung (1993) that, as long as the first order condition is fulfilled, global optimality in continuous time settings without persistence is not

\footnotetext{
${ }^{12}$ We show in Corollary 1 that $\rho>k$, whereas $v<0$ follows from the specification (11) of the utility function.

${ }^{13}$ This will not necessarily be true if we allowed effort to have a cumulative effect, i.e., if we let $\delta$ be negative. Then persistence may even lead to backloaded transfers.
} 

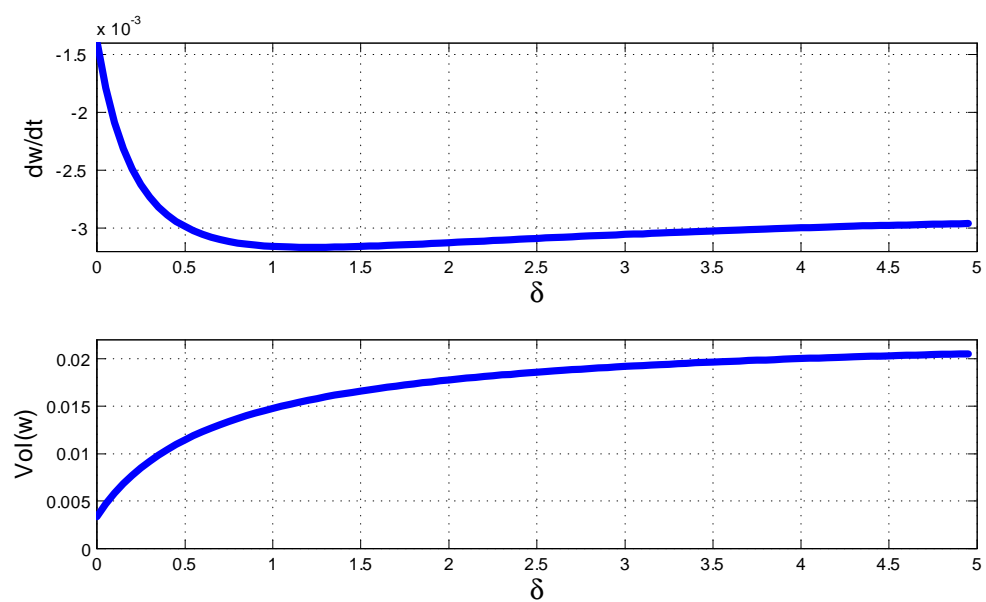

Figure 2: Drift and Volatility of Wages as a function of $\delta$.

Parameters: $\rho=0.1, \lambda=0.5, \sigma=1, \theta=10$.

an issue. ${ }^{14}$ Recent papers by Williams (2011) or Jovanovic and Prat (2013) show that this is not anymore true when actions have a lasting effect. One usually has to impose a bound on the degree of persistence in order to exclude multiple deviations. Interestingly enough, such restrictions are not required in our model, at least when $\delta$ is restricted to positive values so that the effect of effort do not cumulate over time. Determining whether this result is due to the particular structure of our model, or to a more general property arising from the complementarity between effort and persistence, is an interesting issue whose resolution we leave for further research.

Retirement. - The principal may decide to retire the agent instead of paying the compensation required to extract effort. Retirement is profitable when the condition below is satisfied.

Corollary 3 When $1+1 /(\rho+\delta)<\lambda-C$, it is optimal to retire the agent. Otherwise, the contract described in Proposition 3 dominates retirement.

When the condition holds with equality, the cost of compensating the agent for an additional unit of effort equals the expected return. To see why, observe that the cheapest way to deliver the promised value is to pay the constant stream $-\ln (-\rho v) / \theta$. It follows

\footnotetext{
${ }^{14}$ To see that this conclusion holds true in our set-up, set $\delta$ equal to infinity. It follows that $\lim _{\delta \rightarrow \infty} p_{t}=$ $\lim _{\delta \rightarrow \infty} E_{t}\left[\int_{t}^{T} e^{-(\rho+\delta)(s-t)} \gamma_{s} d s\right]=0$. Thus the volatility coefficient $\vartheta_{t}=0$, which implies in turn that the sufficient condition is satisfied since $2 u_{a a}\left(w_{t}, a_{t}\right) \leq 0=\vartheta_{t}$.
} 
from the expression in Proposition 3 that the flow cost of incentive provision with respect to retirement is equal to $\lambda-C$. Effort extraction is profitable solely when this cost is lower than the discounted return of one unit of effort $1+1 /(\rho+\delta)$. Note that Corollary 3 also excludes stopping times as the principal will never provide incentives for a while and then retire the agent.

As one should expect, learning-by-doing renders retirement less attractive. The noise parameter $\sigma$ has the opposite effect because it raises $|C|$. Actually, since $C$ diverges with $\sigma$, there always exists an output variance above which retirement dominates. By contrast, the effect of $\delta$ on $C$ is bounded from below by the solution prevailing in the absence of human capital accumulation.

\section{Conclusion}

We have derived the necessary condition that any dynamic contract has to satisfy when agents learn by doing. A significant benefit of using a continuous time approach is that it also delivers a sufficient condition which ensures that the contract is fully incentive compatible. We showed that, when the agent's utility is CARA, sufficiency is not an issue as long as the impact of effort does not build up over time.

This paper contributes to the burgeoning literature on dynamic contracts with persistent information. Research on such problems is still at an early stage, but recent breakthroughs suggest that it may soon become useful for the analysis of empirical data. For example, our model predicts that income volatility increases when human capital accumulation becomes more difficult or less relevant. Hence, introducing a finite lifetime or a concave learning technology leads to rising wage volatility over the lifecycle, as documented in, e.g., Gibbons and Murphy (1992).

Enriching the model proposed in this paper also promises to improve our understanding of actual contracts. An interesting but demanding extension would combine hidden types with learning-by-doing. In such an environment, actions serve two purposes: Apart from building up future skills, effort establishes the agent's reputation. Both motives are particularly important for young workers. But the companion paper Jovanovic and Prat (2013) shows that career concerns and learning-by-doing have opposite effects on the power of incentives. The tension is likely to differ across occupations and could therefore helps explaining the variety of contractual arrangements observed in reality. 


\section{APPENDIX A}

Proof. Proposition 1: Consider the Brownian motion $Z^{0}$ under some probability space with probability measure $Q$, and let $\mathbb{F}^{Z^{0}} \triangleq\left\{\mathcal{F}_{t}^{Z^{0}}\right\}_{0 \leq t \leq T}$ denote the suitably augmented filtration generated by $Z^{0}$. Let

$$
Y_{t}=\int_{0}^{t} \sigma d Z_{s}^{0}
$$

so that $Y_{t}$ is also a Brownian motion under $Q$. Since expected output is linear in cumulative output, the exponential local martingale

$$
\Lambda_{t, \tau}^{a} \triangleq \exp \left(\int_{t}^{\tau}\left(\frac{h_{s}+a_{s}}{\sigma}\right) d Z_{s}^{0}-\frac{1}{2} \int_{t}^{\tau}\left|\frac{h_{s}+a_{s}}{\sigma}\right|^{2} d s\right)
$$

for $t \leq \tau \leq T$, is a martingale, i.e., $E_{t}\left[\Lambda_{t, T}^{a}\right]=1$. Hence Girsanov theorem holds and ensures that

$$
Z_{t}^{a} \triangleq Z_{t}^{0}-\int_{0}^{t} \frac{h_{s}+a_{s}}{\sigma} d s
$$

is a Brownian motion under the new probability measure $d Q^{a} / d Q \triangleq \Lambda_{0, T}^{a}$. Given that both measures are equivalent, the triple $\left(Y, Z^{a}, Q^{a}\right)$ is a weak solution of the SDE

$$
Y_{t}=\int_{0}^{t}\left(h_{s}+a_{s}\right) d s+\int_{0}^{t} \sigma d Z_{s}^{a}
$$

Adopting a weak formulation allows us to view the choice of control $a$ as determining the probability measure $Q^{a}$. In order to define the agent's optimization problem, let $R^{a}(t)$ denote the reward from time $t$ onwards so that

$$
R^{a}(t) \triangleq e^{\rho t}\left[\int_{t}^{T} u\left(s, Y ., a_{s}\right) d s+U(T, Y .)\right]
$$

where the output path is denoted by $Y$. and, with a slight abuse of notation, $u\left(s, Y ., a_{s}\right) \triangleq$ $e^{-\rho s} u\left(w(Y), a_{s}\right)$ and $U(T, Y$. $) \triangleq e^{-\rho T} U(Y$. $)$ are utilities at time $t$ discounted from time 0 . The agent's objective is to find an admissible control process that maximizes the expected reward $E^{a}\left[R^{a}(0)\right]$ over all admissible controls $a \in \mathcal{A}$. In other words, the agent solves 
the following problem

$$
v_{t}=\sup _{a_{t} \in \mathcal{A}} V^{a}(t) \triangleq \sup _{a_{t} \in \mathcal{A}} E_{t}^{a}\left[R^{a}(t)\right], \text { for all } 0 \leq t \leq T
$$

The objective function can be recast as

$$
V^{a}(t)=E_{t}^{a}\left[R^{a}(t)\right]=E_{t}\left[\Lambda_{t, T}^{a} R^{a}(t)\right],
$$

where the operator $E^{a}[\cdot]$ and $E[\cdot]$ are expectations under the probability measure $Q^{a}$ and $Q$, respectively. One can see from (16) that varying $a$ is indeed equivalent to changing the probability measure. The key advantage of the weak formulation is that, under the reference measure $Q$, the output process does not depend on $a$. Hence, we can treat it as fixed which enables us to solve our problem in spite of its non-Markovian structure.

Our derivation of the necessary conditions builds on the variational argument in Cvitanić et al. (2009). Define the control perturbation

$$
a^{\varepsilon} \triangleq a+\varepsilon \Delta a
$$

We assume that there exists an $\varepsilon_{0}>0$ for which any $\varepsilon \in\left[0, \varepsilon_{0}\right)$ satisfy $\left|a^{\varepsilon}\right|^{4},\left|u^{a^{\varepsilon}}\right|^{4},\left|u_{a}^{a^{\varepsilon}}\right|^{4},\left|\Lambda_{t, \tau}^{a^{\varepsilon}}\right|^{4}$, $\left(\mathcal{U}_{t, \tau}^{a^{\varepsilon}}\right)^{2}$ and $\left(\partial_{a} \mathcal{U}_{t, \tau}^{a^{\varepsilon}}\right)^{2}$ being uniformly integrable in $L^{1}(Q)$ where

$$
\mathcal{U}_{t, \tau}^{a} \triangleq \int_{t}^{\tau} u\left(s, Y ., a_{s}\right) d s
$$

We introduce the following shorthand notations for "variations"

$$
\begin{aligned}
\nabla \mathcal{U}_{t, \tau}^{a} & \triangleq \int_{t}^{\tau} u_{a}\left(s, Y, a_{s}\right) \Delta a_{s} d s \\
\nabla h_{t} & \triangleq \int_{0}^{t} e^{-\delta(t-s)} \Delta a_{s} d s \\
\nabla \Lambda_{t, \tau}^{a} & \triangleq \frac{\Lambda_{t, \tau}^{a}}{\sigma}\left[\int_{t}^{\tau}\left(\nabla h_{s}+\Delta a_{s}\right) d Z_{s}^{0}-\int_{t}^{\tau}\left(h_{t}+a_{s}\right)\left(\nabla h_{s}+\Delta a_{s}\right) d s\right] \\
& =\frac{\Lambda_{t, \tau}^{a}}{\sigma} \int_{t}^{\tau}\left(\nabla h_{s}+\Delta a_{s}\right) d Z_{s}^{a} .
\end{aligned}
$$


Step 1: We first characterize the variations of the agent's objective with respect to $\varepsilon$

$$
\begin{aligned}
\frac{V^{a^{\varepsilon}}(t)-V^{a}(t)}{\varepsilon} & =E\left[\Lambda_{t, T}^{a^{\varepsilon}} R^{a^{\varepsilon}}(t)-\Lambda_{t, T}^{a} R^{a}(t)\right] \\
& =E\left[\left(\frac{\Lambda_{t, T}^{a^{\varepsilon}}-\Lambda_{t, T}^{a}}{\varepsilon}\right) R^{a^{\varepsilon}}(t)+\Lambda_{t, T}^{a}\left(\frac{R^{a^{\varepsilon}}(t)-R^{a}(t)}{\varepsilon}\right)\right] \\
& =E\left[\nabla \Lambda_{t, T}^{a^{\varepsilon}} R^{a^{\varepsilon}}(t)+\Lambda_{t, T}^{a}\left(\frac{R^{a^{\varepsilon}}(t)-R^{a}(t)}{\varepsilon}\right)\right] .
\end{aligned}
$$

To obtain the limit of the first term as $\varepsilon$ goes to zero, observe that

$$
\nabla \Lambda_{t, T}^{a^{\varepsilon}} R^{a^{\varepsilon}}(t)-\nabla \Lambda_{t, T}^{a} R^{a}(t)=\left[\nabla \Lambda_{t, T}^{a^{\varepsilon}}-\Lambda_{t, T}^{a}\right] R^{a}(t)+\nabla \Lambda_{t, T}^{a^{\varepsilon}}\left[R^{a^{\varepsilon}}(t)-R^{a}(t)\right] .
$$

As shown in Cvitanić et al. (2009), for any $\varepsilon \in\left[0, \varepsilon_{0}\right)$, this expression is integrable uniformly with respect to $\varepsilon$ and so

$$
\lim _{\varepsilon \rightarrow 0} E\left[\nabla \Lambda_{t, T}^{a^{\varepsilon}} R^{a^{\varepsilon}}(t)\right]=E\left[\nabla \Lambda_{t, T}^{a} R^{a}(t)\right]
$$

The limit of the second term reads

$$
\lim _{\varepsilon \rightarrow 0} \frac{R^{a^{\varepsilon}}(t)-R^{a}(t)}{\varepsilon}=e^{\rho t} \nabla \mathcal{U}_{t, T}^{a} .
$$

Due to the uniform integrability of $\Lambda_{t, T}^{a}\left(R^{a^{\varepsilon}}(t)-R^{a}(t)\right) / \varepsilon$, the expectation is also well defined. Combining the two expressions above, we finally obtain

$$
\lim _{\varepsilon \rightarrow 0} \frac{V^{a^{\varepsilon}}(t)-V^{a}(t)}{\varepsilon}=E\left[\nabla \Lambda_{t, T}^{a} R^{a}(t)+\Lambda_{t, T}^{a} e^{\rho t} \nabla \mathcal{U}_{t, T}^{a}\right] \triangleq \nabla V^{a}(t)
$$

Step 2: We are now in a position to derive the necessary condition. Consider total earnings as of date 0

$$
I^{a}(t) \triangleq E_{t}^{a}\left[\int_{0}^{T} u\left(s, Y ., a_{s}\right) d s+U(T, Y .)\right]=\int_{0}^{t} u\left(s, Y ., a_{s}\right) d s+e^{-\rho t} V^{a}(t) .
$$

By definition, it is a $Q^{a}$-martingale. According to the extended Martingale Representation Theorem ${ }^{15}$ of Fujisaki et al. (1972), all square integrable $Q^{a}$-martingales are

\footnotetext{
${ }^{15}$ We cannot directly apply the standard Martingale Representation theorem because we are considering weak solutions, so that $\left\{Z_{t}^{a}\right\}$ does not necessarily generate $\left\{\mathcal{F}_{t}^{Y}\right\}$.
} 
stochastic integrals of $\left\{Z_{t}^{a}\right\}$ and there exists a unique process $\zeta$ in $L^{2}\left(Q^{a}\right)$ such that

$$
I^{a}(T)=I^{a}(t)+\int_{t}^{T} \zeta_{s} \sigma d Z_{s}^{a}
$$

This decomposition allows us to solve for $\nabla V^{a}(t)$. Reinserting (17), (18) and (19) into (20) yields $^{16}$

$$
\begin{aligned}
\nabla V^{a}(t) & =E_{t}\left[R^{a}(t) \frac{\Lambda_{t, T}^{a}}{\sigma} \int_{t}^{T}\left(\nabla h_{s}+\Delta a_{s}\right) d Z_{s}^{a}+\Lambda_{t, T}^{a} e^{\rho t} \int_{t}^{T} u_{a}(\cdot) \Delta a_{s} d s\right] \\
& =e^{\rho t} E_{t}^{a}\left[\frac{I^{a}(T)}{\sigma} \int_{t}^{T}\left(\nabla h_{s}+\Delta a_{s}\right) d Z_{s}^{a}+\int_{t}^{T} u_{a}(\cdot) \Delta a_{s} d s\right] .
\end{aligned}
$$

where subscripts denote derivatives and arguments are omitted for brevity. Given the law of motion (22), applying Ito's rule to the first term yields

$$
\begin{aligned}
d\left(\frac{I^{a}(\tau)}{\sigma} \int_{t}^{\tau}\left(\nabla h_{s}+\Delta a_{s}\right) d Z_{s}^{a}\right)= & {\left[\zeta_{\tau}\left(\nabla h_{\tau}+\Delta a_{\tau}\right)\right] d \tau } \\
& +\left[\zeta_{\tau} \int_{t}^{\tau}\left(\nabla h_{s}+\Delta a_{s}\right) d Z_{s}^{a}+I_{t}^{a}(\tau)\left(\nabla h_{\tau}+\Delta a_{\tau}\right)\right] d Z_{\tau}^{a} .
\end{aligned}
$$

Hence $\nabla V^{a}(t)$ can be represented as

$$
e^{-\rho t} \nabla V^{a}(t)=E_{t}^{a}\left[\int_{t}^{T} \Gamma_{s}^{1} d s+\int_{t}^{T} \Gamma_{s}^{2} d Z_{s}^{a}\right]
$$

where

$$
\begin{aligned}
& \Gamma_{s}^{1} \triangleq \zeta_{s}\left[\int_{0}^{s} e^{-\delta(s-\tau)} \Delta a_{\tau} d \tau+\Delta a_{s}\right]+u_{a}\left(s, Y ., a_{s}\right) \Delta a_{s}, \\
& \Gamma_{s}^{2} \triangleq \zeta_{s}\left[\int_{t}^{s}\left(\int_{0}^{\tau} e^{-\delta(\tau-r)} \Delta a_{r} d r+\Delta a_{\tau}\right) d Z_{\tau}^{a}\right]+I_{t}^{a}(s)\left(\int_{0}^{s} e^{-\delta(s-\tau)} \Delta a_{\tau} d \tau+\Delta a_{s}\right) .
\end{aligned}
$$

Given that $\Gamma_{s}^{2}$ is square integrable, ${ }^{17}$ we have $E_{t}^{a}\left[\int_{t}^{T} \Gamma_{s}^{2} d Z_{s}^{a}\right]=0$. As for the deterministic

${ }^{16}$ The additional expectation term vanishes because both $\nabla A_{s}$ and $\Delta a_{s}$ are bounded and so

$$
\left(\int_{0}^{t} U\left(\tau, Y, a_{\tau}\right) d \tau\right) E_{t}^{a}\left[\int_{t}^{T}\left(\nabla A_{s}+\Delta a_{s}\right) d Z_{s}^{a}\right]=0 .
$$

${ }^{17}$ Square integrability of $\Gamma_{s}^{2}$ can be established for any $\varepsilon \in\left[0, \varepsilon_{0}\right)$ following the same steps as in Lemma 7.3 of Cvitanic et al. (2009). 
term, collecting the effect of each perturbation $\Delta a_{s}$ yields

$$
e^{-\rho t} \nabla V^{a}(t)=E_{t}^{a}\left[\int_{t}^{T}\left(\int_{s}^{T} e^{-\delta(\tau-s)} \zeta_{\tau} d \tau+\zeta_{s}+u_{a}\left(s, Y ., a_{s}\right)\right) \Delta a_{s} d s\right] .
$$

Finally, noticing that $\Delta a_{s}$ was arbitrary, optimality of $a_{t}$ requires that

$$
\left(E_{t}^{a}\left[\int_{t}^{T} e^{-\delta(s-t)} \zeta_{s} d s\right]+\zeta_{t}+u_{a}\left(t, Y, a_{t}\right)\right)\left(a-a_{t}\right) \leq 0
$$

We now rewrite the necessary condition as a function of the volatility $\gamma$ of the promised value $v_{t}$. Differentiating $(21)$ with respect to time yields

$$
d I^{a}(t)=e^{-\rho t}\left[d v_{t}-\rho v_{t}+u\left(Y, a_{t}\right)\right]=\zeta_{t} \sigma d Z_{t}^{a}
$$

so that

$$
d v_{t}=\left(\rho v_{t}-u\left(Y, a_{t}\right)\right) d t+e^{\rho t} \zeta_{t} \sigma d Z_{t}^{a}
$$

Replacing $\gamma_{t}=e^{\rho t} \zeta_{t}$ into (23) and collecting the exponential terms, one obtains the necessary condition (6).

Proof. Proposition 2: We wish to compare rewards along the equilibrium path $\left\{a_{t}^{*}\right\}_{t=0}^{T}$ with those derived following an arbitrary strategy $\left\{a_{t}\right\}_{t=0}^{T}$. As in the proof of Proposition 1 let $\Delta a_{t} \triangleq a_{t}-a_{t}^{*}$ denote the local effort deviation. Similarly, let

$$
\nabla h_{t} \triangleq h_{t}-h_{t}^{*}=\int_{0}^{t} e^{-\delta(t-s)} \Delta a_{s} d s
$$

denote the resulting differences in human capital at each date. The Brownian motions generated by the two effort policies satisfy

$$
\sigma d Z_{t}^{a^{*}}=\sigma d Z_{t}^{a}+\left[a_{t}+h_{t}-\left(a_{t}^{*}+h_{t}^{*}\right)\right] d t=\sigma d Z_{t}^{a}+\left(\Delta a_{t}+\nabla h_{t}\right) d t
$$

The total rewards $I^{a^{*}}(t)$ from strategy $a$ as of date 0 can therefore be decomposed as follows

$$
\begin{aligned}
I^{a^{*}}(T) & =\int_{0}^{T} e^{-\rho t} u\left(Y ., a_{t}\right) d t+e^{-\rho T} U(Y .)=V^{a^{*}}(0)+\int_{0}^{T} e^{-\rho t} \gamma_{t}^{*} \sigma d Z_{t}^{a^{*}} \\
& =V^{a^{*}}(0)+\int_{0}^{T} e^{-\rho t} \gamma_{t}^{*}\left(\Delta a_{t}+\nabla h_{t}\right) d t+\int_{0}^{T} e^{-\rho t} \gamma_{t}^{*} \sigma d Z_{t}^{a}
\end{aligned}
$$


Hence, the total reward from the arbitrary policy is given by

$$
\begin{aligned}
I^{a}(T) & =\int_{0}^{T} e^{-\rho t}\left[u\left(Y, a_{t}\right)-u\left(Y ., a_{t}^{*}\right)\right] d t+I^{a^{*}}(T) \\
& =\int_{0}^{T} e^{-\rho t}\left[u\left(Y ., a_{t}\right)-u\left(Y ., a_{t}^{*}\right)\right] d t+V^{a^{*}}(0)+\int_{0}^{T} e^{-\rho t} \gamma_{t}^{*}\left(\Delta a_{t}+\nabla h_{t}\right) d t+\int_{0}^{T} e^{-\rho t} \gamma_{t}^{*} \sigma d Z_{t}^{a} .
\end{aligned}
$$

Let us focus on the third term on the right hand side

$$
\begin{aligned}
\int_{0}^{T} e^{-\rho t} \gamma_{t}^{*} \nabla h_{t} d t & =\int_{0}^{T} e^{-\rho t} \gamma_{t}^{*}\left(\int_{0}^{t} e^{-\delta(t-s)} \Delta a_{s} d s\right) d t=\int_{0}^{T} e^{\delta t} \Delta a_{t}\left(\int_{t}^{T} e^{-(\delta+\rho) s} \gamma_{s}^{*} d s\right) d t \\
& =\int_{0}^{T} e^{\delta t} \Delta a_{t}\left[e^{-(\delta+\rho) t} p_{t}^{*}+\int_{t}^{T} e^{-(\delta+\rho) s} \vartheta_{s}^{*} \sigma d Z_{s}^{a^{*}}\right] d t
\end{aligned}
$$

where the last equality follows from the definition of $p$ and $\vartheta$. Changing the Brownian motion in the last term on the RHS and taking expectation yields

$$
\begin{aligned}
E_{0}^{a}\left[\int_{0}^{T} e^{\delta t} \Delta a_{t}\left(\int_{t}^{T} e^{-(\delta+\rho) s} \vartheta_{s}^{*} \sigma d Z_{s}^{a^{*}}\right) d t\right] & =E_{0}^{a}\left[\int_{0}^{T} e^{\delta t} \Delta a_{t}\left(\int_{t}^{T} e^{-(\delta+\rho) s} \vartheta_{s}^{*}\left(\Delta a_{s}+\nabla h_{s}\right) d s\right) d t\right] \\
& =E_{0}^{a}\left[\int_{0}^{T} e^{-\rho t} \vartheta_{t}^{*}\left(\Delta a_{t}+\nabla h_{t}\right)\left(\int_{0}^{t} e^{-\delta(t-s)} \Delta a_{s}\right) d t\right] \\
& =E_{0}^{a}\left[\int_{0}^{T} e^{-\rho t} \vartheta_{t}^{*}\left(\Delta a_{t}+\nabla h_{t}\right) \nabla h_{t} d t\right] .
\end{aligned}
$$

Hence we have

$$
V^{a}(0)-V^{a^{*}}(0)=E_{0}^{a}\left[\int_{0}^{T} e^{-\rho t}\left(u\left(w_{t}, a_{t}\right)-u\left(w_{t}, a_{t}^{*}\right)+\left(\gamma_{t}^{*}+p_{t}^{*}\right) \Delta a_{t}+\vartheta_{t}^{*}\left(\Delta a_{t}+\nabla h_{t}\right) \nabla h_{t}\right) d t\right] .
$$

We know from the optimization property of $a_{t}^{*}$ that the first expectation term is at most equal to zero. On the other hand, the sign of the second expectation term is ambiguous. In order to bound it, we introduce the predictable process ${ }^{18} \chi_{t}^{*} \triangleq \gamma_{t}^{*}-\vartheta_{t}^{*} h_{t}^{*}$ and define the function

$$
H\left(a, h ; \chi^{*}, \vartheta^{*}, p^{*}\right) \triangleq u(w, a)+\left(\chi^{*}+\vartheta^{*} h\right) a+\vartheta^{*} h^{2}+p^{*} a .
$$

\footnotetext{
${ }^{18} \chi^{*}$ is predictable since both $\xi^{*}$ and $h^{*}$ are $\mathbb{F}^{Y}$-predictable.
} 
Taking a linear approximation of $H(\cdot)$ around $A^{*}$ yields

$$
\begin{aligned}
& H\left(a_{t}, h_{t}\right)-H\left(a_{t}^{*}, h_{t}^{*}\right)-\frac{\partial H\left(a_{t}^{*}, h_{t}^{*}\right)}{\partial h} \nabla h_{t} \\
= & u\left(w_{t}, a_{t}\right)-u\left(w_{t}, a_{t}^{*}\right)+\left(\chi_{t}^{*}+\vartheta_{t}^{*} h_{t}^{*}\right) \Delta a_{t}+a_{t} \vartheta_{t}^{*}\left(h_{t}-h_{t}^{*}\right) \\
& +\vartheta_{t}^{*}\left(h_{t}^{2}-h_{t}^{* 2}\right)+p_{t}^{*} \Delta a_{t}-\left[\vartheta_{t}^{*} a_{t}^{*}+2 \vartheta_{t}^{*} h_{t}^{*}\right] \nabla h_{t} \\
= & u\left(w_{t}, a_{t}\right)-u\left(w_{t}, a_{t}^{*}\right)+\left(\gamma_{t}^{*}+p_{t}^{*}\right) \Delta a_{t}+\vartheta_{t}^{*} \nabla h_{t}\left(a_{t}-a_{t}^{*}\right)+\vartheta_{t}^{*}\left(h_{t}^{2}-h_{t}^{* 2}-2 \Delta_{t} h_{t}^{*}\right) \\
= & u\left(w_{t}, a_{t}\right)-u\left(w_{t}, a_{t}^{*}\right)+\left(\gamma_{t}^{*}+p_{t}^{*}\right) \Delta a_{t}+\vartheta_{t}^{*} \nabla h_{t}\left(\Delta a_{t}+\nabla h_{t}\right) .
\end{aligned}
$$

The expected benefits of following an alternative strategy can therefore be written as

$$
V^{a}(0)-V^{a^{*}}(0)=E_{0}^{a}\left[\int_{0}^{T} e^{-\rho t}\left(H\left(a_{t}, h_{t}\right)-H\left(a_{t}^{*}, h_{t}^{*}\right)-\frac{\partial H\left(a_{t}^{*}, h_{t}^{*}\right)}{\partial h} \nabla h_{t}\right) d t\right]
$$

which is negative when $H(\cdot)$ is jointly concave. Given that the agent seeks to maximize expected returns, imposing concavity ensures that $a^{*}$ dominates any alternative effort path. Concavity is established considering the Hessian matrix of $H(\cdot)$

$$
\mathcal{H}(t, a, h)=\left(\begin{array}{cc}
u_{a a}\left(w_{t}, a_{t}\right) & \vartheta_{t} \\
\vartheta_{t} & 2 \vartheta_{t}
\end{array}\right)
$$

which is negative semi-definite when $2 u_{a a}\left(w_{t}, a_{t}\right) \leq \vartheta_{t}$, as stated in (10).

Proof. Proposition 3: In order to simplify the algebra, we start by focusing on contracts which extract full effort. Then we can omit actions $a$ from the list of control and recast the principal's optimization problem as

$$
B_{t}=\min _{\{w, \gamma, \vartheta\}} E_{t}^{a}\left[\int_{t}^{\infty} e^{-\rho(s-t)} w_{s} d s\right],
$$

subject to

$$
\begin{aligned}
d v_{t} & =\left[\rho v_{t}-u\left(w_{t}, 1\right)\right] d t+\gamma_{t} \sigma d Z_{t} \\
d p_{t} & =\left[(\rho+\delta) p_{t}-\gamma_{t}\right] d t+\vartheta_{t} \sigma d Z_{t} \\
\gamma_{t} & =-u_{a}\left(w_{t}, 1\right)-p_{t}
\end{aligned}
$$

i.e., the two promise-keeping constraints and the necessary condition under which income volatility is minimized. 
The Hamilton-Jacobi-Bellman (HJB hereafter) equation associated to the principal's optimal control problem reads ${ }^{19}$

$$
\rho B(v, p)=\min _{\{w, \vartheta\}}\left\{\begin{array}{c}
w+\frac{\partial B}{\partial v}(\rho v-u(w, 1))+\frac{\partial B}{\partial p}((\rho+\delta) p-\gamma) \\
+\frac{\sigma^{2}}{2}\left[\frac{\partial^{2} B}{\partial v^{2}} \gamma(p, w)^{2}+\frac{\partial^{2} B}{\partial p^{2}} \vartheta^{2}+2 \frac{\partial^{2} B}{\partial v \partial p} \gamma(p, w) \vartheta\right]
\end{array}\right\}
$$

where we have used (26) to express $\gamma$ as a function of $p$ and $w$. We seek a solution to the HJB equation of the following form

$$
\rho B(v)=\lambda-\frac{\ln (-\rho v)}{\theta}-C
$$

and guess that the optimal wage schedule is equal to

$$
w(v)=-\frac{\ln (-k v)}{\theta}+\lambda \Rightarrow u(w(v), 1)=k v
$$

while the value of private information is given by

$$
p(v)=-\theta \lambda \frac{k}{k+\delta+1} v
$$

Under our premise that $p$ is equal to $v$ times a constant, we can eliminate $p$ from the HJB equation and rewrite it as follows

$$
\rho B(v)=\min _{w}\left\{w+B^{\prime}(v)(\rho v-u(w, 1))+\frac{\sigma^{2}}{2} B^{\prime \prime}(v) \gamma(v, w)^{2}\right\} .
$$

The FOC with respect to wages reads

$$
1-B^{\prime}(v) u_{w}(w, 1)+\sigma^{2} B^{\prime \prime}(v) \gamma(v, w) \frac{\partial \gamma(v, w)}{\partial w}=0
$$

In order to obtain the expression of $\partial \gamma / \partial w$, we replace our guess into (26) to obtain

$$
\gamma=-u_{a}(w(v), 1)-p=-\theta \lambda\left(k-\frac{k}{k+\delta+1}\right) v=-\theta \lambda k\left(\frac{k+\delta}{k+\delta+1}\right) v
$$

\footnotetext{
${ }^{19}$ In our particular problem, switching from a weak formulation of the agent's problem, to a strong formulation of the principal's problem, does not raise measurability issues because the agent's action is constant over time, and so does not directly depends on the Brownian motion.
} 
Differentiating this expression with respect to wages yields

$$
\frac{\partial \gamma(v, w)}{\partial w}=-u_{a w}(w(v), 1)=-\theta \gamma+\theta^{2} \lambda \frac{k}{k+\delta+1} v
$$

The FOC (32) for wages is therefore equivalent to

$1+B^{\prime}(v) \theta v k-\sigma^{2} B^{\prime \prime}(v) \theta^{3}(\lambda v)^{2}\left[\left(k-\frac{k}{k+\delta+1}\right)^{2}+\left(k-\frac{k}{k+\delta+1}\right) \frac{k}{k+\delta+1}\right]=0$, or

$$
\rho-k-(\theta \lambda \sigma)^{2}\left[k\left(k-\frac{k}{k+\delta+1}\right)\right]=\rho-k-(\theta \lambda \sigma)^{2} k^{2}\left(\frac{k+\delta}{k+\delta+1}\right)=0 .
$$

Thus $k$ solves the following cubic equation

$$
k^{3}(\theta \lambda \sigma)^{2}+k^{2}\left[(\theta \lambda \sigma)^{2} \delta+1\right]+k(\delta+1-\rho)-\rho(\delta+1)=0
$$

The relevant solution is given by the positive root because wages are not defined when $k$ is negative. The existence and uniqueness of $k$ are established below in the proof of Corollary 1.

We now verify that the dynamic programming equation is indeed satisfied

$$
\begin{aligned}
\rho B(v) & =w+B^{\prime}(v)(\rho v-u(w(v), 1))+B^{\prime \prime}(v) \frac{\sigma^{2}}{2} \gamma^{2} \\
& =\lambda-\frac{\ln (-k v)}{\theta}-\frac{\rho-k}{\rho \theta}+\frac{(\sigma \lambda)^{2} \theta}{2 \rho}\left[k\left(\frac{k+\delta}{k+\delta+1}\right)\right]^{2}
\end{aligned}
$$

Replacing our guess for $B(v)$ on the left hand side, one finds that the HJB holds true for all promised value $v$ as long as

$$
C=\frac{\rho-k}{\rho \theta}-\frac{(\sigma \lambda)^{2} \theta}{2 \rho}\left[k\left(\frac{k+\delta}{k+\delta+1}\right)\right]^{2}
$$

We still have to verify our guess (30) for $p$. Reinserting the Incentive Constraint (26) into the law of motion of $p$, we find that

$$
d p_{t}=\left[(\rho+\delta+1) p_{t}+u_{a}\left(w_{t}, 1\right)\right] d t+\vartheta_{t} \sigma d Z_{t}
$$


Integrating this expression with respect to time yields

$$
p_{t}=-E\left[\int_{t}^{\infty} e^{-(\rho+\delta+1)(s-t)} u_{a}\left(w_{s}, 1\right) d s\right]
$$

which, using our specification (11) of the utility function and wage function (29), yields

$$
p_{t}=-\theta \lambda E_{t}\left[\int_{t}^{\infty} e^{-(\rho+\delta+1)(s-t)} u\left(w_{s}, 1\right) d s\right]=-\theta \lambda \int_{t}^{\infty} e^{-(\rho+\delta+1)(s-t)} k E_{t}\left[v_{s}\right] d s
$$

The expression of $E_{t}\left[v_{s}\right]$ follows from the law of motion of $v$

$$
d v_{t}=\left[\rho v_{t}-u\left(w_{t}, 1\right)\right] d t+\gamma_{t} \sigma d Z_{t}=v_{t}(\rho-k) d t+\gamma_{t} \sigma d Z_{t}
$$

Since $\gamma_{t}$ is square integrable, we have $E_{t}\left[v_{s}\right]=v_{t} \exp ((\rho-k)(s-t))$. Replacing this expression into (35), we finally obtain

$$
p_{t}=-\theta \lambda k v_{t} \int_{t}^{\infty} e^{-(k+\delta+1)(s-t)} d s=-\theta \lambda \frac{k}{k+\delta+1} v_{t}
$$

as conjectured in (30).

The last step consists in establishing that the contract maximizes the principal's profit. First notice that the profit function $\pi$ can be rewritten as follows

$$
\begin{aligned}
\pi\left(h_{t}, v_{t}\right) & =\max _{\{a, w, \gamma, \vartheta\}} E_{t}\left[\int_{t}^{\infty} e^{-\rho(s-t)}\left(a_{s}+h_{s}-w_{s}\right) d s\right] \\
& =h_{t} \int_{t}^{\infty} e^{-(\rho+\delta)(s-t)} d s+\max _{\{a, w, \gamma, \vartheta\}} E_{t}\left[\int_{t}^{\infty} e^{-\rho(s-t)}\left(a_{s}+\int_{t}^{s} e^{-\delta(s-\tau)} a_{\tau} d \tau-w_{s}\right) d s\right] \\
& =\frac{h_{t}}{\rho+\delta}+\pi\left(0, v_{t}\right) .
\end{aligned}
$$

Since the agent's incentive constraint is independent of the level of human capital, neither the principal's objective function nor its constraint depend on $h$, and Bellman's principle of optimality implies that it is equivalent to maximize $\pi\left(0, v_{t}\right)$ or $\pi\left(h_{t}, v_{t}\right)$. We can therefore rewrite the principal problem as

$$
\pi\left(v_{t}\right)=\max _{\{a, w, \gamma, \vartheta\}} E_{t}\left[\int_{t}^{\infty} e^{-\rho(s-t)}\left(a_{s}\left[1+\frac{1}{\rho+\delta}\right]-w_{s}\right) d s\right]
$$

subject to $(24),(25)$ and (26). According to the steps above, our guess for the value 
function

$$
\rho \pi(v)=1+\frac{1}{\rho+\delta}-\rho B(v)
$$

is a solution of the dynamic programming equation

$$
\rho \pi(v)=\sup _{\{a, w\}}\left\{a\left(1+\frac{1}{\rho+\delta}\right)-w+\pi^{\prime}(v)(\rho v-u(w, a))+\pi^{\prime \prime}(v) \frac{\sigma^{2}}{2} \gamma(v, w, a)^{2}\right\} .
$$

Furthermore, for each fixed $v \in \mathbb{R}$, the supremum with respect to wages is attained when $w(v)=-\ln (-k v) / \theta+\lambda$. For optimal effort to be equal to one, we must have

$$
1+\frac{1}{\rho+\delta}+B^{\prime}(v) u_{a}(w, 1)-B^{\prime \prime}(v) \sigma^{2} \gamma(v, w, 1) \frac{\partial \gamma(v, w, 1)}{\partial a} \geq 0
$$

To see that the inequality is indeed satisfied, differentiate the incentive constraint (26) to obtain $\partial \gamma / \partial a=-\lambda \partial \gamma / \partial w$. Reinserting this equality along with $u_{a}(\cdot)=-\lambda u_{w}(\cdot)$ into the FOC (32) for wages, we find that (37) is strictly positive. Hence the supremum with respect to effort is attained when $a=1$, which completes the verification argument.

Proof. Corollary 1: The first step consists in characterizing the cubic equation defining $k$

$$
k^{3}(\theta \lambda \sigma)^{2}+k^{2}\left[(\theta \lambda \sigma)^{2} \delta+1\right]+k(\delta+1-\rho)-\rho(\delta+1)=0 .
$$

Observe that it can be rewritten as follows

$$
\underbrace{(\theta \lambda \sigma)^{2} k^{2}-\rho}_{\triangleq L(k)}=\underbrace{\frac{k^{2}\left[(\theta \lambda \sigma)^{2}-1\right]-(\delta+1) k}{k+\delta+1}}_{\triangleq R(k)}
$$

We distinguish two cases:

1. $\theta \lambda \sigma \leq 1$ : The function $R(k)$ on the RHS of (39) is decreasing for all $k \in \mathbb{R}^{+}$and $R(0)=0$. By contrast, the function $L(k)$ on the LHS of (39) is always increasing for all $k \in \mathbb{R}^{+}$. Furthermore, since $L(0)=-\rho$ and $\lim _{k \rightarrow \infty} L(k)=\infty$, (39) always admits a unique positive real solution.

2. $\theta \lambda \sigma>1$ : The function $R(k)$ on the RHS of (39) is not anymore decreasing for all $k \in \mathbb{R}^{+}$. However, $R(0)>L(0)$ still holds true and since $\lim _{k \rightarrow \infty} R(k)<\lim _{k \rightarrow \infty} L(k)$, (39) always admits a positive real solution. Furthermore, these two inequalities imply that the number of positive solutions of (39) cannot be equal to two. This means that 


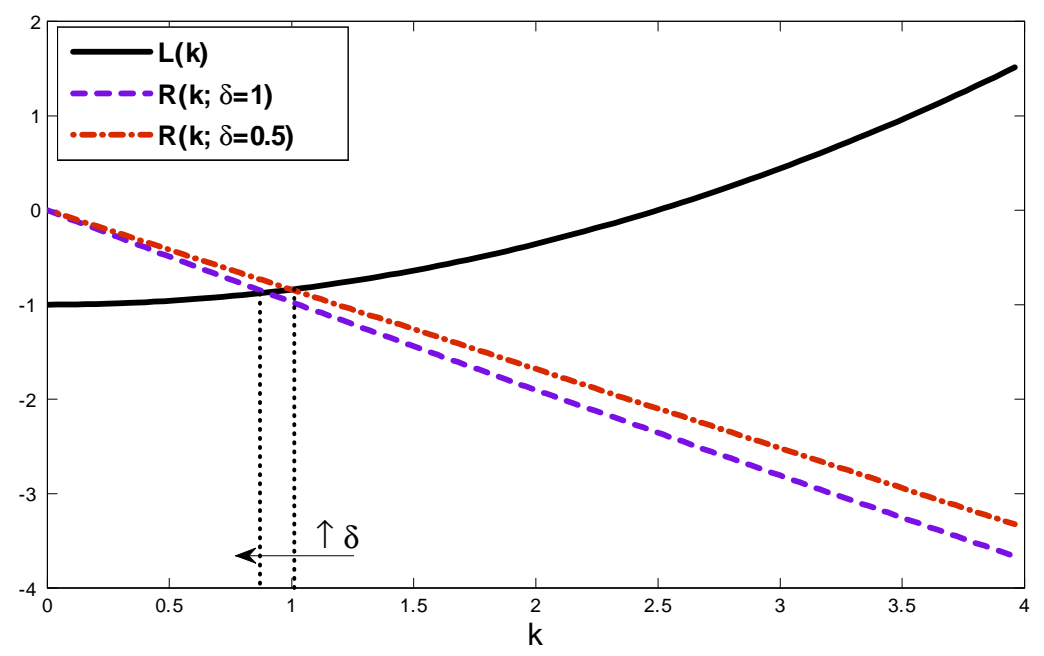

Figure 3: Positive root of the cubic equation (39) for different values of $\delta$.

either one root or all three of them are positive real numbers. But the last possibility being impossible too, ${ }^{20}$ the only remaining option is that (39) has a unique positive real solution.

Having established the uniqueness of $k$, we proceed by showing that $\partial k / \partial \delta<0$. Again, this is most easily established considering (39). Given that $\partial L(k) / \partial \delta=0$ while $\partial R(k) / \partial \delta=-k^{2}(\theta \lambda \sigma)^{2}(k+\delta+1)^{-2}<0$, the solution to (39) must be decreasing in $\delta$. Figure 3 illustrates the comparative statics exercise.

Finally it will prove useful to show that $k<\rho$. Let $\bar{k}$ denote the positive root of (38) when $\delta=0$, i.e.,

$$
\bar{k}^{3}(\theta \lambda \sigma)^{2}+\bar{k}^{2}+\bar{k}(1-\rho)-\rho=0 .
$$

Since $\partial k / \partial \delta<0$ and $\delta \geq 0$, we have $k<\bar{k}$. Assume for the sake of contradiction that $\bar{k}>\rho$, then $\bar{k}^{2}-\bar{k} \rho>0$ and $\bar{k}^{3}(\theta \lambda \sigma)^{2}+\bar{k}^{2}+\bar{k}(1-\rho)>\bar{k}^{3}(\theta \lambda \sigma)^{2}+\bar{k}>\rho$, which contradicts the definition in (40) of $\bar{k}$. Hence it holds true that $\rho>\bar{k}>k$ for all $\delta \geq 0$.

We can finally prove Corollary 1 . Differentiating the incentive $\operatorname{costs} C$ with respect to

\footnotetext{
${ }^{20}$ To establish this claim analytically, one can differentiate $L(k)$ and $R(k)$ to obtain: $L^{\prime \prime}(k)=(\theta \lambda \sigma)^{2}$ and $R^{\prime \prime}(k)=2[\theta \lambda \sigma(\delta+1)]^{2}(k+\delta+1)^{-3}$. Hence there exists a unique $\hat{k}$ such that $L^{\prime \prime}(k) \gtrless R^{\prime \prime}(k)$ for all $k \gtrless \hat{k}$, which rules out the possibility that $R(k)$ intersects twice $L(k)$ from above.
} 
$k$, we get $^{21}$

$$
\begin{aligned}
\frac{\partial C}{\partial k} & =\frac{1}{\theta k}-\frac{1}{\theta \rho}-\frac{(\sigma \lambda)^{2} \theta}{2 \rho} 2\left[k\left(\frac{k+\delta}{k+\delta+1}\right)\right] \frac{\partial}{\partial k}\left[k\left(\frac{k+\delta}{k+\delta+1}\right)\right] \\
& =\frac{1}{\theta}\left[\frac{1}{k}-\frac{1}{\rho}\right]+\frac{(\sigma \lambda)^{2} \theta}{2 \rho} 2 \frac{1}{(\theta \lambda \sigma)^{2}}\left[\frac{\rho}{k}-1\right] \frac{1}{(\theta \lambda \sigma)^{2}} \frac{\rho}{k^{2}}>0
\end{aligned}
$$

The positive sign follows from $k<\rho$, and the corollary holds true since

$$
\frac{\partial C}{\partial \delta}=\underbrace{\frac{\partial C}{\partial k} \frac{\partial k}{\partial \delta}}_{+}-\frac{(\sigma \lambda)^{2} \theta}{2 \rho} k^{2} 2\left(\frac{k+\delta}{k+\delta+1}\right) \frac{1}{(k+\delta+1)^{2}}<0 .
$$

Proof. Corollary 2: The sufficiency condition derived in Proposition 2 is satisfied when $2 u_{a a}\left(w_{t}, 1\right) \leq \vartheta_{t}$. The volatility coefficient $\vartheta$ of $p$ follows from our finding in the proof of Proposition 3 that $p_{t}=-v_{t} \theta \lambda k /(k+\delta+1)$. Applying Ito's lemma, we get

$$
\vartheta_{t}=-\theta \lambda \frac{k}{k+\delta+1} \gamma_{t}=\left(\frac{\theta \lambda k}{k+\delta+1}\right)^{2}(k+\delta) v_{t}
$$

We have also shown that $u\left(w_{t}, 1\right)=k v_{t}$. Given that $u_{a a}\left(w_{t}, 1\right)=(\theta \lambda)^{2} u\left(w_{t}, 1\right)$, the sufficient condition is equivalent to

$$
2 u_{a a}\left(w_{t}, 1\right) \leq \vartheta_{t} \Longleftrightarrow 2 \geq \frac{k(k+\delta)}{(k+\delta+1)^{2}},
$$

which is true for all $\delta \geq 0$.

Proof. Corollary 3: We first show that it is optimal to retire the agent when $1+1 /(\rho+\delta)<\lambda-C$. Let $R(v)$ denote the discounted cost of retiring an agent with promised value $v$. Since there is no need to motivate the agent, the principal perfectly insures her; in other words $\gamma_{t}=0$ and $d v_{t} / d t=\rho\left(v_{t}\right)-u\left(w_{t}, 0\right)=0$ for all $t \geq 0$.

${ }^{21}$ The second equality below follows from the definition of $k$ as

$$
k\left(\frac{k+\delta}{k+\delta+1}\right)=\frac{\rho-k}{(\theta \lambda \sigma)^{2} k} .
$$


Evaluating the principal's value function $R$ associated to retirement yields

$$
\rho R\left(v_{t}\right)=-\rho \int_{t}^{\infty} e^{-\rho(s-t)} w\left(v_{t}\right) d s=-w\left(v_{t}\right)=\frac{\ln (-\rho v)}{\theta} .
$$

Retirement at date 0 dominates incentive provision when

$$
R(v)-\pi(0, v)=-1-\frac{1}{\rho+\delta}-C+\lambda>0,
$$

as stated in Corollary 3. We still have to prove that there is no optimal stopping rule such that retirement follows a period with effort exertion. We have shown in the proof of Proposition 3 that

$$
\pi_{t}(v)=\frac{h_{t}}{\delta(\rho+\delta)}+\pi_{0}(v)=\frac{1-e^{-\delta t}}{\delta(\rho+\delta)}+\pi_{0}(v),
$$

where we have used the fact that $h$ is deterministic to substitute it with $t$. Thus, if the agent is not immediately retired at date 0 , we must have $0>R(v)-\pi_{0}(v)>R(v)-\pi_{t}(v)$, showing that it can never be optimal to retire the agent at a future date. 


\section{APPENDIX B}

The closed-from solution described in Proposition 3 holds when the contracting horizon is infinite. We show in this Appendix that the same expression can be obtained by solving for fully incentive contracts with a finite horizon and then letting the retirement date go to infinity.

As in the main text, we restrict our attention to the exponential utility functions

$$
u(w, a)=-\exp (-\theta(w-\lambda a)), \text { with } \lambda \in(0,1), \theta>0
$$

with $a \in[0,1]$. Since the agent continuation value is given by

$$
v_{t} \triangleq \max _{a_{t} \in \mathcal{A}} E_{t}^{a}\left[\int_{t}^{T} e^{-\rho(s-t)} u\left(w_{s}, a_{s}\right) d s+e^{-\rho(T-t)} U\left(W_{T}\right)\right]
$$

we also have to specify the termination utility $U(W)$. We assume that it lies on the Pareto frontier under zero effort, i.e.,

$$
U(W)=-\frac{\exp (-\theta \rho W)}{\rho}
$$

This is equivalent to assuming that agents are infinitely lived and that they retire at date $T$. While retired, they consume the perpetual annuity derived from $W$ and provide zero effort. Aside from its natural interpretation, this specification has been chosen for its tractability since the particular form of $U(W)$ will become immaterial to our results as we shall let the contracting horizon diverge to infinity.

Proposition 4 Assume that: (i) $u(w, a)$ and $U(W)$ are as specified in (42) and (43);

(ii) Recommended effort is set equal to its first-best level $a_{t}^{*}=1$. The expected wage bill as a function of the current date $t$ and of the retirement date $T$ reads

$$
\rho B^{T}(t, v)=f^{T}(t)-\frac{\ln (-\rho v)}{\theta}
$$

The function $f^{T}(t)$ is given by

$$
f^{T}(t)=-\int_{t}^{T} e^{-\rho(s-t)}\left[\rho\left(\frac{\ln \left(k_{t}^{T} / \rho\right)}{\theta}-\lambda\right)-\frac{(\sigma \lambda)^{2} \theta}{2}\left(\left(\varphi_{s}^{T}\right)^{2}-\left(k_{s}^{T}\right)^{2}\right)\right] d s,
$$


where $k_{t}^{T}$ is the positive solution of

$$
\rho-k_{t}^{T}-(\theta \lambda \sigma)^{2}\left[k_{t}^{T}\left(k_{t}^{T}-\varphi_{t}^{T}\right)\right]=0, \text { with } \varphi_{t}^{T}=\int_{t}^{T} e^{-\int_{t}^{s}\left(k_{\tau}^{T}+\delta+1\right) d \tau} k_{s}^{T} d s
$$

Furthermore, when $T$ goes to infinity, $B^{T}(\cdot)$ converges pointwise to

$$
\lim _{T \rightarrow \infty} B^{T}(t, v)=B(v)=\lambda-\frac{\ln (-\rho v)}{\theta}-C
$$

where $C$ is defined in Proposition 3.

Proof. Proposition 4: Step 1: Initial guess.-We seek a solution to the HJB equation of the following form

$$
\rho b^{T}(t, v)=f_{t}^{T}-\frac{\ln (-\rho v)}{\theta}
$$

and guess that the associated wage schedule and value of private information are given by

$$
\begin{aligned}
& w_{t}(v)=-\frac{\ln \left(-k_{t}^{T} v\right)}{\theta}+\lambda \Rightarrow u\left(w_{t}(v), 1\right)=k_{t}^{T} v \\
& p_{t}(v)=-\theta \lambda \varphi_{t}^{T} v
\end{aligned}
$$

where $f_{t}^{T}, k_{t}^{T}$ and $\varphi_{t}^{T}$ are continuously differentiable functions that depends solely on time $t$. We impose the boundary condition $\rho b^{T}(T, v)=-\ln (-\rho v) / \theta$.

Step 2: Incentive Constraint.-According to our guess, the Incentive Constraint reads

$$
\gamma_{t}=-u_{a}\left(w_{t}(v), a_{t}\right)-p_{t}=-\theta \lambda\left(k_{t}^{T}-\varphi_{t}^{T}\right) v_{t}
$$

Differentiating it with respect to wages yields

$$
\frac{\partial \gamma(t, v, w, a)}{\partial w}=-u_{a w}\left(w_{t}(v), a_{t}\right)=-\theta\left(\gamma_{t}-\theta \lambda \varphi_{t}^{T} v_{t}\right)
$$

The FOC (32) for wages is therefore equivalent to

$$
1+\frac{\partial b^{T}(t, v)}{\partial v} \theta k_{t}^{T} v-\sigma^{2} \frac{\partial^{2} b^{T}(t, v)}{\partial v^{2}} \theta^{3}(\lambda v)^{2}\left[k_{t}^{T}\left(k_{t}^{T}-\varphi_{t}^{T}\right)\right]=0
$$


Replacing the derivatives of $b^{T}(\cdot)$ with respect to $v$, we obtain the characteristic equation

$$
\rho-k_{t}^{T}-(\theta \lambda \sigma)^{2}\left[k_{t}^{T}\left(k_{t}^{T}-\varphi_{t}^{T}\right)\right]=0
$$

The relevant solution $k_{t}^{T}$ is given by the positive root because wages are not defined when $k_{t}^{T}$ is negative.

Step 3: HJB equation.-The dynamic programming equation reads

$$
\begin{aligned}
\rho b^{T}(t, v) & =w_{t}(v)+\frac{\partial b^{T}(t, v)}{\partial t}+\frac{\partial b^{T}(t, v)}{\partial v}\left(\rho v-u\left(w_{t}(v), 1\right)\right)+\frac{\partial^{2} b^{T}(t, v)}{\partial v^{2}} \frac{\sigma^{2}}{2} \gamma^{2} \\
& =\lambda-\frac{\ln \left(-k_{t}^{T} v\right)}{\theta}+\frac{1}{\rho} \frac{d f^{T}(t)}{d t}-\frac{\rho-k_{t}^{T}}{\rho \theta}+\frac{(\sigma \lambda)^{2} \theta}{2 \rho}\left(k_{t}^{T}-\varphi_{t}^{T}\right)^{2} .
\end{aligned}
$$

The HJB is satisfied for all promised value $v$ when

$$
\frac{d f^{T}(t)}{d t}-\rho f^{T}(t)=\rho\left(\frac{\ln \left(k_{t}^{T} / \rho\right)}{\theta}-\lambda\right)-\frac{(\sigma \lambda)^{2} \theta}{2}\left(\left(\varphi_{t}^{T}\right)^{2}-\left(k_{t}^{T}\right)^{2}\right),
$$

or

$$
f^{T}(t)=-\int_{t}^{T} e^{-\rho(s-t)} \underbrace{\left[\rho\left(\frac{\ln \left(k_{t}^{T} / \rho\right)}{\theta}-\lambda\right)-\frac{(\sigma \lambda)^{2} \theta}{2}\left(\left(\varphi_{s}^{T}\right)^{2}-\left(k_{s}^{T}\right)^{2}\right)\right]}_{\triangleq \psi_{s}^{T}} d s .
$$

Step 4: Verification of the guess for $p_{t}$.-Following the steps leading to equation (35), we find that

$$
p_{t}=-E_{t}\left[\int_{t}^{T} e^{-(\rho+\delta+1)(s-t)} u_{a}\left(w_{s}, a_{s}\right) d s\right]=-\theta \lambda \int_{t}^{T} e^{-(\rho+\delta+1)(s-t)} k_{s}^{T} E_{t}\left[v_{s}\right] d s .
$$

The SDE for $v_{t}$ reads

$$
d v_{t}=v_{t}\left[\left(\rho-k_{t}^{T}\right) d t-\Gamma_{t}^{T} \sigma d Z_{t}\right]
$$

where $\Gamma_{t}^{T} \triangleq \theta \lambda\left(k_{t}^{T}-\varphi_{t}^{T}\right)$. Whenever $\Gamma_{t}^{T}$ is bounded, a conjecture that will be justified below, we have $E_{t}\left[v_{s}\right]=v_{t} \exp \left(\int_{t}^{s}\left(\rho-k_{\tau}^{T}\right) d \tau\right)$ and

$$
p_{t}=-\theta \lambda \underbrace{\left(\int_{t}^{T} e^{-\int_{t}^{s}\left(k_{\tau}^{T}+\delta+1\right) d \tau} k_{s}^{T} d s\right)}_{=\varphi_{t}^{T}} v_{t}
$$


Given the definition of $k_{t}^{T}$, the coefficient $\varphi_{t}^{T}$ is positive and bounded which implies in turn that, as conjectured above, $\Gamma_{t}^{T}$ is bounded. Hence, we have verified our guess for the functional form of $p_{t}$. Note that the value of private information $p_{T}=\varphi_{T}^{T}=0$ because earnings are constant after date $T$.

Step 5: Verification theorem.-Our guess for the value function $b^{T} \in \mathcal{C}^{1,2}([0, T[\times \mathbb{R}) \cap$ $\mathcal{C}([0, T] \times \mathbb{R})$ is a solution of the dynamic programming equation

$\rho b^{T}(t, v)=\frac{\partial b^{T}(t, v)}{\partial t}+\inf _{w}\left\{w+\frac{\partial b^{T}(t, v)}{\partial v}(\rho v-u(w, 1))+\frac{\partial^{2} b^{T}(t, v)}{\partial v^{2}} \frac{\sigma^{2}}{2} \gamma(t, v, w, 1)^{2}\right\}$

with boundary condition $\rho b^{T}(T, v)=-\ln (-\rho v) / \theta$. Furthermore, for each fixed $(t, v) \in$ $[0, T[\times \mathbb{R}$, the infimum in the expression

$$
\inf _{w}\left\{w+\frac{\partial b^{T}(t, v)}{\partial v}(\rho v-u(w, 1))+\frac{\partial^{2} b^{T}(t, v)}{\partial v^{2}} \frac{\sigma^{2}}{2} \gamma(t, v, w, 1)^{2}\right\}
$$

is attained by $w^{*}(t, v)=-\ln \left(-k_{t}^{T} v\right) / \theta+\lambda$. Thus the verification theorem holds and the value function for the control problem $B^{T}(t, v)=b^{T}(t, v)$ while $w^{*}(t, v)$ is the optimal markovian control.

Step 6: Incentive compatibility.-The sufficient condition $2 u_{a a}\left(w_{t}, 1\right) \leq \vartheta_{t}$ is satisfied when $2 \geq \varphi_{t}^{T}\left(1-\varphi_{t}^{T} / k_{t}^{T}\right)$. Since both $\varphi_{t}^{T}$ and $k_{t}^{T}$ are positive, $2 u_{a a}\left(w_{t}, 1\right) \leq \vartheta_{t}$ whenever $\varphi_{t}^{T} \leq 1$. To show that this restriction holds true, note that $\partial \varphi_{t}^{T} / \partial T=$ $k_{t}^{T}-\varphi_{t}^{T}\left(k_{t}^{T}+\delta+1\right)$. This differential equation implies that $\partial \varphi_{t}^{T} / \partial T=0$ whenever $\varphi_{t}^{T}=k_{t}^{T} /\left(k_{t}^{T}+\delta+1\right)$. Given that the definition (48) of $k_{t}^{T}$ is such that $\partial k_{t}^{T} / \partial T=0$ when $\partial \varphi_{t}^{T} / \partial T=0$, we have $\partial^{2} \varphi_{t}^{T} / \partial T^{2}=\partial \varphi_{t}^{T} / \partial T=0$ whenever $\varphi_{t}^{T}=k_{t}^{T} /\left(k_{t}^{T}+\delta+1\right)$. This shows that $\varphi_{t}^{T}$ is bounded, either from above or below, by $k_{t}^{T} /\left(k_{t}^{T}+\delta+1\right)$. But we know that $\varphi_{t}^{t}=0$. Hence it must be the case that $\varphi_{t}^{T} \leq k_{t}^{T} /\left(k_{t}^{T}+\delta+1\right)<1$ and, as explained before, the sufficient condition (10) is fulfilled.

Step 7: Convergence as $T$ goes to infinity.-The solutions for $k_{t}^{T}$ and $\varphi_{t}^{T}$ are found by backward induction using the terminal condition $\varphi_{T}^{T}=0$ and reinserting the expression for $\varphi_{t}^{T}$ into the quadratic equation (48) defining $k_{t}^{T}$. Let $k \triangleq \lim _{T \rightarrow \infty} k_{t}^{T}$ so that $\varphi \triangleq$ $\lim _{T \rightarrow \infty} \varphi_{t}^{T}=k /(k+\delta+1)$. Reinserting the expression of $\varphi_{t}^{T}$ into (48), we find that $k$ is indeed given by the positive solution of the cubic equation (14). The pointwise convergence of $k_{t}^{T}$ and $\varphi_{t}^{T}$ ensure that $\psi_{t}^{T}$ converges pointwise to

$$
\psi \triangleq \lim _{T \rightarrow \infty} \psi_{t}^{T}=\rho\left(\frac{\ln (k / \rho)}{\theta}-\lambda\right)-\frac{(\sigma \lambda)^{2} \theta}{2}\left(\left(\frac{k}{k+\delta+1}\right)^{2}-k^{2}\right) .
$$


Applying the dominated convergence theorem to evaluate the limit of the function $f_{t}^{T}$ defined in (50), we obtain

$$
\begin{aligned}
\lim _{T \rightarrow \infty} f_{t}^{T} & =-\int_{t}^{T} e^{-\rho(s-t)} \psi d s=\lambda-\frac{\ln (k / \rho)}{\theta}+\frac{(\sigma \lambda)^{2} \theta}{2 \rho}\left(\left(\frac{k}{k+\delta+1}\right)^{2}-k^{2}\right) \\
& =\lambda-\frac{\ln (k / \rho)}{\theta}-\frac{\rho-k_{t}^{T}}{\rho \theta}+\frac{(\sigma \lambda)^{2} \theta}{2 \rho}\left[k\left(\frac{k}{k+\delta+1}\right)\right]^{2} .
\end{aligned}
$$

Given that $\lim _{T \rightarrow \infty} f_{t}^{T}=\lambda-C$, we have $\lim _{T \rightarrow \infty} B^{T}(t, v)=B(v)$ and the value function described in Proposition 3 is indeed the limit of a sequence of finite horizon problems.

\section{References}

[1] Becker, Gary. Human Capital: A Theoretical and Empirical Analysis, with Special Reference to Education. Chicago, University of Chicago Press, 1964.

[2] Cvitanić, Jakša, Wan, Xuhu and Jianfeng Zhang. "Optimal Compensation with Hidden Action and Lump-Sum Payment in a Continuous-Time Model." Applied Mathematics and Optimization 59 (2009): 99-146.

[3] DeMarzo, Peter M. and Yuliy Sannikov. "Learning in Dynamic Incentive Contracts." Princeton University University, Unpublished manuscript (2011).

[4] Fernandes, Ana and Christopher Phelan. "A Recursive Formulation for Repeated Agency with History Dependence." Journal of Economic Theory 91 (2000): 223-247.

[5] Fujisaki, Masatoshi, Kallianpur, Gopinath and Hiroshi Kunita. "Stochastic Differential Equation for the Non Linear Filtering Problem." Osaka Journal of Mathematics 9 (1972):19-40.

[6] Gibbons, Robert and Kevin J. Murphy. "Optimal Incentive Contracts in the Presence of Career Concerns: Theory and Evidence." Journal of Political Economy 100(3) (1992): 468-505.

[7] He, Zhiguo, Wei, Bin and Jianfeng Yu. "Optimal Long-term Contracting with Learning."University of Chicago, Unpublished manuscript (2012). 
[8] Jovanovic, Boyan, and Julien Prat. "Dynamic Contracts when Agent's Quality is Unknown." Forthcoming in Theoretical Economics.

[9] Sannikov, Yuliy. "A Continuous-Time Version of the Principal-Agent Problem." Review of Economic Studies, 75(3) (2008): 957-984.

[10] Sannikov, Yuliy. "Moral Hazard and Long-Run Incentives." Princeton University, Unpublished manuscript (2012).

[11] Schättler, Heinz and Jaeyoung Sung. "The First-Order Approach to the ContinuousTime Principal-Agent Problem with Exponential Utility." Journal of Economic Theory 61 (1993): 331-371.

[12] Williams, Noah. "Persistent Private Information." Econometrica 79 (2011): 12331274.

[13] Williams, Noah. "On Dynamic Principal-Agent Problems in Continuous Time." University of Wisconsin - Madison, Unpublished manuscript (2013). 